\title{
Estimaciones del consumo de drogas ilicitas derivadas del análisis de aguas residuales: Una revisión crítica
}

\author{
Illicit drug consumption estimations derived from \\ wastewater analysis: A critical review
}

\begin{abstract}
Aldo Pacheco-Ferreira ${ }^{1}$
Forma de citar: Pacheco-Ferreira A. Estimaciones del consumo de drogas ilícitas derivadas del análisis de aguas residuales: Una revisión crítica. Rev Univ Ind Santander Salud. 2019; 51(1): 69-80. doi: http://dx.doi.org/10.18273/revsal.v51n1-2019008 (c) (i)
\end{abstract}

\section{Resumen}

La medición cuantitativa de drogas ilícitas en aguas residuales puede proporcionar información objetiva sobre los patrones y tendencias del uso de psicotrópicos en la comunidad. Se analizó la presencia de psicoactivos en plantas de tratamiento para aguas residuales y en el medio ambiente por epidemiología de alcantarillado, mediante una revisión sistemática de 2008-2017 por medio de los descriptores controlados: "drogas ilícitas", "plantas de tratamiento de aguas residuales" y "ambiente" hecha en las bases de datos Medline vía PubMed, SciELO, BVS vía LILACS y Google Académico. La búsqueda arrojó 439 estudios y fueron seleccionados 34 estudios para análisis. Los años de publicación variaron, siendo 2016 el de mayor de publicaciones (diez), seguido de 2014 y 2012 (cinco), 2017, 2013, 2010 (tres), 2011 (dos), 2015, 2009 y 2008 (uno cada uno). Los resultados mostraron la presencia de indicadores químicos de las drogas clásicas cocaína y cannabis. La investigación sobre la presencia de drogas ilícitas, particularmente como compuestos activos en el medio ambiente, es vital para mejorar el conocimiento de los tipos consumidos, su prevalencia; siendo capaz desplegar para analizar la exposición y el impacto de estos compuestos en el medio ambiente y la salud pública. Los resultados de esta revisión han resaltado que la epidemiología basada en aguas residuales es un enfoque útil y poderoso.

Palabras clave: Drogas ilícitas; Ambiente; Planta de tratamiento de aguas residuales; Revisión.

\begin{abstract}
Quantitative measurement of illicit drugs in wastewater can provide objective information on community drug use patterns and trends. The presence of illicit drugs in treatment plants for wastewater and in the environment was analyzed by sewege epidemiology. It is a systematic review from 2008-2017 by means of the controlled descriptors: "illicit drugs", "wastewater treatment plants" and "environment" performed on Medline databases via PubMed, SciELO, BVS via LILACS and Google Scholar. The search yielded 439 studies. 34 studies were selected for analysis. The years of publication varied, with 2016 being the highest of publications (ten), followed by 2014 and 2012 (five),

1. Escuela Nacional de Salud Pública Sérgio Arouca/Fiocruz. Río de Janeiro, Brasil.

Correspondencia: Aldo Pacheco Ferreira. Dirección: Departamento de Derechos Humanos, Salud y Diversidad Cultural (DIHS/ENSP/ FIOCRUZ). Avenida Brasil, 4036, sala 905. Manguinhos - Rio de Janeiro - RJ, Cep: 21.041-361. Teléfono: +55 21 $3882-9222$. Correo electrónico: aldopachecoferreira@gmail.com.
\end{abstract}


2017, 2013, 2010 (three), 2011 (two), 2015, 2009 and 2008 (one each). The results showed the presence of chemical indicators of the classic drugs cocaine and cannabis. Research on the presence of illicit drugs, particularly as active compounds in the environment, are vital to improve knowledge of the types consumed, its occurrence; being able to unfold to analyze the exposure and the impact of these compounds in the environment and public health. The results of this revision have highlighted that wastewater-based epidemiology is a useful and powerful approach.

Keywords: Illicit drugs; Environment; Wastewater treatment plants; Review.

\section{Introducción}

El uso de drogas ilícitas existe en diferentes partes del mundo, determinando en cada cultura múltiples funciones y patrones de consumo ${ }^{1,2}$. Sin embargo, a pesar del carácter permanente de esta práctica humana, a partir del siglo XX se vivió un escenario en el que el abuso de psicotrópicos se configuró como un problema social exigiendo medidas específicas de diferentes sectores sociales y gubernamentales ${ }^{3,4}$.

Debido al impacto social que genera el abuso de psicoactivos es importante analizar efectos y prácticas políticas derivadas, a partir de la articulación y mediación de factores de orden individual, sociocultural y político-económico 5 . Por lo tanto, en virtud de las consecuencias del uso en la vida de las personas y comunidades, así como de los prejuicios y estigmas vigentes, se acompaña la inserción de esta temática en la agenda de las políticas públicas, teniendo en cuenta el papel destacado del sector salud ${ }^{6,7}$.

El cannabis y la cocaína son las drogas ilícitas de mayor consumo ${ }^{8,9}$. La cocaína (COC) es una sustancia alcaloide obtenida de las hojas del arbusto Erytroxylon coca, cuyos metabolitos principales son: la benzoilecgonina (BE) y la ecgoninametilester (EME). La planta es originaria de los países de la región andina de América. Su nombre proviene de una de las culturas más desarrolladas del Alto Perú, la aimará, en donde se le llamaba KkoKa, que significa arbusto ${ }^{10}$.

La marihuana es el nombre dado a las hojas y flores secas de la planta Cannabis sativa, preparada como una mezcla para fumar; en otro estado, hachís es la resina extraída. La planta se utiliza desde hace siglos para fines recreacionales y medicinales. Contiene en promedio 400 sustancias químicas y al menos 60 alcaloides conocidos como cannabinoides. Entre ellos, el tetrahidrocannabinol (THC) es el más activo y principal responsable de los efectos producidos ${ }^{11}$.

Los psicoactivos se convirtieron en objeto de investigación socio-antropológica tardíamente ${ }^{12}$. En la primera mitad del siglo $\mathrm{XX}$, lo que fue producido de forma disciplinaria, como investigación sociológica académicamente establecida, tuvo un carácter eminentemente accesorio, como forma de complementación de los esfuerzos médicos y sanitarios en entender y resolver los problemas relacionados al consumo de drogas: dependencia, crimen y disgregación social ${ }^{13}$.

Con la universalización y generalización de los psicotropicos, la comprensión del consumo asumió dos perspectivas científicas: una cualitativa, en la cual el consumo es visto como la pérdida de la exclusividad de su uso por parte de ciertos grupos y clases sociales, otra cuantitativa, que se presenta en mayor prevalencia, o sea, de utilización cotidiana y con tendencia a la aceptación social ${ }^{14,15}$.

Esta dinámica de consumo caracterizada por la normalización o el alojamiento de las drogas, se refiere a la banalización de su presencia y la disminución de la percepción del peligro, resultando por así decir, en una facilitación del uso/abuso y de los riesgos a la salud. Esta lógica favoreció la expansión de las drogas, principalmente las ilegales, a mediados de la década de $1990^{8}$.

En consecuencia, este fenómeno debe ser visto como un proceso social macroestructural, configurándose en una cuestión de salud pública y de seguridad nacional, suscitando la debida atención a su complejidad. Diversos factores se encuentran asociados a este fenómeno, y se cree, que la dificultad en los análisis se debe a la calidad y disponibilidad de la informacion que tal vez favorece la desinformación o la contrainformación, produciendo saturación funcional y ocultación de sus problemas reales ${ }^{16}$.

Las consecuencias del consumo frecuente de drogas son determinantes para la morbimortalidad, pero no siempre bien dimensionados por la población. Hay actitudes ambivalentes y estigmatizantes entre su uso y usuario, presentes en las diversas culturas, variando en intensidad de acuerdo a múltiples factores: tipo de droga, sexo, edad, clase social y tiempo de uso. En el caso de las drogas ilícitas se suma, el carácter ilegal del uso y la implicación del tráfico ${ }^{12}$. 
Las drogas ilícitas y sus metabolitos son contaminantes emergentes cuyo impacto para los ecosistemas es escasamente conocido ${ }^{17-19}$. Entran a la red de aguas residuales como drogas inalteradas y/o sus metabolitos activos por excreción humana, saliva, y sudor ${ }^{20}$. Sin embargo, ha habido un creciente interés en el estudio de estas sustancias debido a su elevado consumo y continua descarga en compartimentos medioambientales, tras su insuficiente eliminación en las plantas de tratamiento para aguas residuales (PTAR), en donde son removidos y/o biodegradados parcialmente, quedando concentraciones traza en sus efluentes, contaminando así las aguas superficiales y suelos ${ }^{21}$.

La epidemiología basada en análisis de aguas residuales o también llamada epidemiología de alcantarilla constituye una herramienta importante para la estimación del consumo local a través de la investigación de los flujos de masa de drogas de abuso inalteradas o de sus metabolitos ${ }^{22}$. Consecuentemente, la naturaleza y cantidad de estos contaminantes están frecuentemente relacionados con las características, estado de salud y hábitos de las poblaciones que vierten a estas PTARs. Por todo ello, el estudio de estos contaminantes tiene una doble vertiente: epidemiológicamente se pueden evaluar la magnitud, naturaleza y patrones de consumo a través del abanico de compuestos detectados en las PTARs y ambientalmente se puede seguir el rastro y gestionar el peligro que estas sustancias representan para los ecosistemas.

Así, consiste en varios pasos consecutivos que permiten a los investigadores identificar y cuantificar la cantidad de drogas ilícitas correspondientes, que habría sido consumido por la población atendida por la PTAR.

Sin embargo, los hallazgos de dicho análisis están sujetos a incertidumbres, principalmente asociados con el muestreo, el análisis de biomarcadores y la estabilidad, el cálculo retroactivo del consumo de drogas y la estimación del tamaño de la población ${ }^{23}$. Según EMCDDA ${ }^{24}$ esfuerzos para minimizar los posibles errores y estandarizar todos los procedimientos han tenido éxito. La adopción de un procedimiento estandarizado también mejorará la credibilidad y la escalabilidad de los estudios al garantizar que los datos de diferentes fuentes sean más confiables y comparables.

Trabajos recientes han buscado establecer un nuevo enfoque para estimar de forma rápida $\mathrm{y}$ segura el consumo de drogas de abuso. En este contexto, el presente trabajo presenta una revisión sistemática en el tema, con el aporte de un método actual y de extrema relevancia, posibilitando identificar trabajos de investigación y resultados de estudios empíricos que buscaron evaluar la presencia de drogas ilícitas en el alcantarillado. La detección del consumo de drogas de abuso tiene interés en variadas situaciones, con destaque para las de carácter clínico o forense, ya que constituye un serio problema de salud pública.

\section{Metodología}

Se trata de una revisión sistemática que difiere de la revisión tradicional, buscando superar posibles sesgos en todas las etapas, siguiendo un método riguroso de búsqueda y selección de investigación; evaluación de la relevancia y validez de las encontradas; colecta, síntesis e interpretación de sus datos ${ }^{25}$.

Se adoptó un conjunto de criterios que determinan la cientificidad de una revisión sistemática de la literatura, iniciando por la construcción de un protocolo, para garantizar el rigor del proceso de investigación. Para ello, el protocolo disponía de los siguientes componentes: pregunta de revisión, criterios de inclusión y exclusión, estrategias para la búsqueda del universo de investigaciones, orientación para la selección del material, análisis y síntesis de los datos.

La pregunta elaborada para la revisión actual fue: ¿Cuál es la situación real sobre la detección drogas ilícitas y metabólitos en PTARs, en el contexto de la epidemiología de alcantarillado?

Se realizó una búsqueda estratégica de enero de 2008 a Diciembre de 2017. La búsqueda se efectuó en las bases de datos científicas: Medline - Medical Literature Analysis and Retrieval System Online vía PubMed, SciELO - Scientific Electronic Library Online, BVS - Biblioteca Virtual de Salud vía LILACS y Google Académico, empleando los descriptores controlados: "drogas ilícitas", "plantas de tratamiento de aguas residuales" y "ambiente". Además, se utilizó el operador AND.

Los criterios de inclusión fueron: artículos publicados entre 2008 y 2017 que utilizaran la epidemiología de alcantarillado para detectar drogas ilícitas en PTARs $y$ en el medio ambiente, escritos en castellano, inglés o portugués; mientras que los de exclusión fueron: estudios experimentales, estudios que no investigaron drogas ilícitas, estudios farmacológicos y los estudios que abordaron drogas diferentes a la cocaína y la marihuana. 
Para la definición de los artículos seleccionados, fue realizada la lectura de los resúmenes y de los artículos completos por dos investigadores. Se aplicaron las instrucciones provistas por Moher, et al. ${ }^{26}$ en Preferred Reporting Items for Systematic Reviews and MetaAnalyses: The PRISMA Statement. Una vez identificada la literatura elegible, se exporto a Mendeley, un gerente de referencia, para organizar títulos por autor y año de publicación, excluyendo duplicados. Los estudios elegibles se leyeron en texto completo y los datos relevantes fueron extraídos. Las listas de referencias también fueron evaluadas. Finalmente, los datos cuantitativos y cualitativos fueron segregados en categorías para análisis.

Es importante resaltar que el instrumento propuesto es fundamental para la construcción de la revisión, en base a los datos recolectados, fue posible realizar la caracterización de las publicaciones incluidas en el estudio, así como la interpretación y el análisis de los hallazgos y la síntesis del conocimiento producido, siendo expuesta en tablas, de acuerdo con los enfoques temáticos establecidos.

\section{Resultados}

\section{Categorización de las producciones localizadas}

Para esta revisión sistemática, la búsqueda de literatura electrónica y gris arrojó un total de 439 documentos. Fueron excluidos 308 estudios por no relacionarse con los objetivos de la investigación. 97 estudios fueron excluidos por duplicidad en las bases de datos. 34 estudios cumplieron con los criterios de inclusión, se revisaron las listas de referencias seleccionadas sin incluir más estudios. La síntesis del resultado de la búsqueda realizada se encuentra sistematizada en el diagrama de flujo (Figura 1).

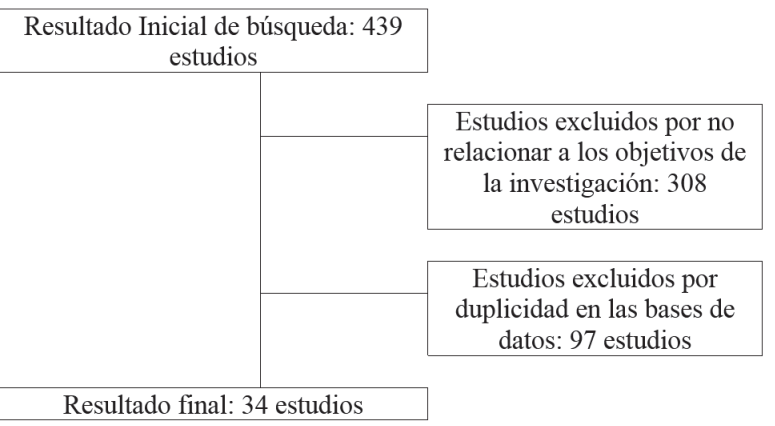

Figura 1. Flujograma del proceso de búsqueda y selección de los estudios de investigación.

\section{Descripción y análisis de los artículos}

Los años de publicación variaron de 2008 a 2017, siendo 2016 el de mayor de publicaciones (diez), seguido de 2014 y 2012 (cinco), 2017, 2013, 2010 (tres), 2011 (dos), 2015, 2009 y 2008 (uno cada uno). Fueron extraídos de los textos analizados los siguientes datos: autor, lugar, método/objetivo y conclusiones (Tabla 1).

Con los informes del consumo de COC de los artículos fue posible calcular que tanto la COC como su principal metabolito urinario, la $\mathrm{BE}$, el consumo promedio en torno a 230 (mg/día)/(1000 personas). Esto equivale a, aproximadamente dos dosis diarias entre 1000 personas, suponiendo que la dosis de referencia de COC es de $100 \mathrm{mg}^{52}$, para el metabolito del cannabis varía entre 108 - 133 (mg/día)/(1000 personas) $)^{34-36,38-40,41,44,45,48,56-58}$.

Las concentraciones de BE, COC y THC medidas en los afluentes de la PTAR se basan en los resultados antes mencionados, una estimación del consumo de cocaína se llevó a cabo teniendo en cuenta el enfoque sugerido por Zuccato, et al. ${ }^{18}$. La idea básica considera que la concentración de $\mathrm{BE}$ en agua $\left(\mathrm{ng} \mathrm{L}^{-1}\right)$ de muestras de aguas residuales debe multiplicarse por 2,33 para correlacionarse directamente con la base libre cocaína consumida. Este enfoque tiene en cuenta la relación de masa molar COC/BE de base libre (1.048) y la fracción molar promedio (45\%) de una dosis de cocaína que se excreta como BE, según diferentes estudios.

Se percibe a través de los estudios seleccionados que el uso de drogas es influenciado por una serie de factores, incluyendo características sociodemográficas: edad, sexo, educación, región, y tipos de drogas utilizadas ${ }^{1,6,7,16}$. La mayoría de los estudios, se refieren a las poblaciones estudiantiles y revelan un aumento significativo del uso de las drogas, debido a la vulnerabilidad de estos usuarios, la gran demanda como el hecho de que la mayoría son hombres, con edades entre 20 y 30 años, solteros y desempleados, pero se percibe un aumento relativo del uso de drogas también en el sexo femenino.

\section{Fuentes y distribución en el medio ambiente}

La presencia de drogas ilícitas y sus metabolitos en el ambiente ha sido confirmada desde final del siglo XX, cuando Viana, et al. ${ }^{60}$ verificaron la existencia de cocaína en niveles mensurables en el aire. Sin embargo, sólo a principios de este siglo, estudios sobre la ocurrencia de estas drogas en el ambiente, principalmente en aguas naturales y residuales urbanas, se tornaron frecuentes. 


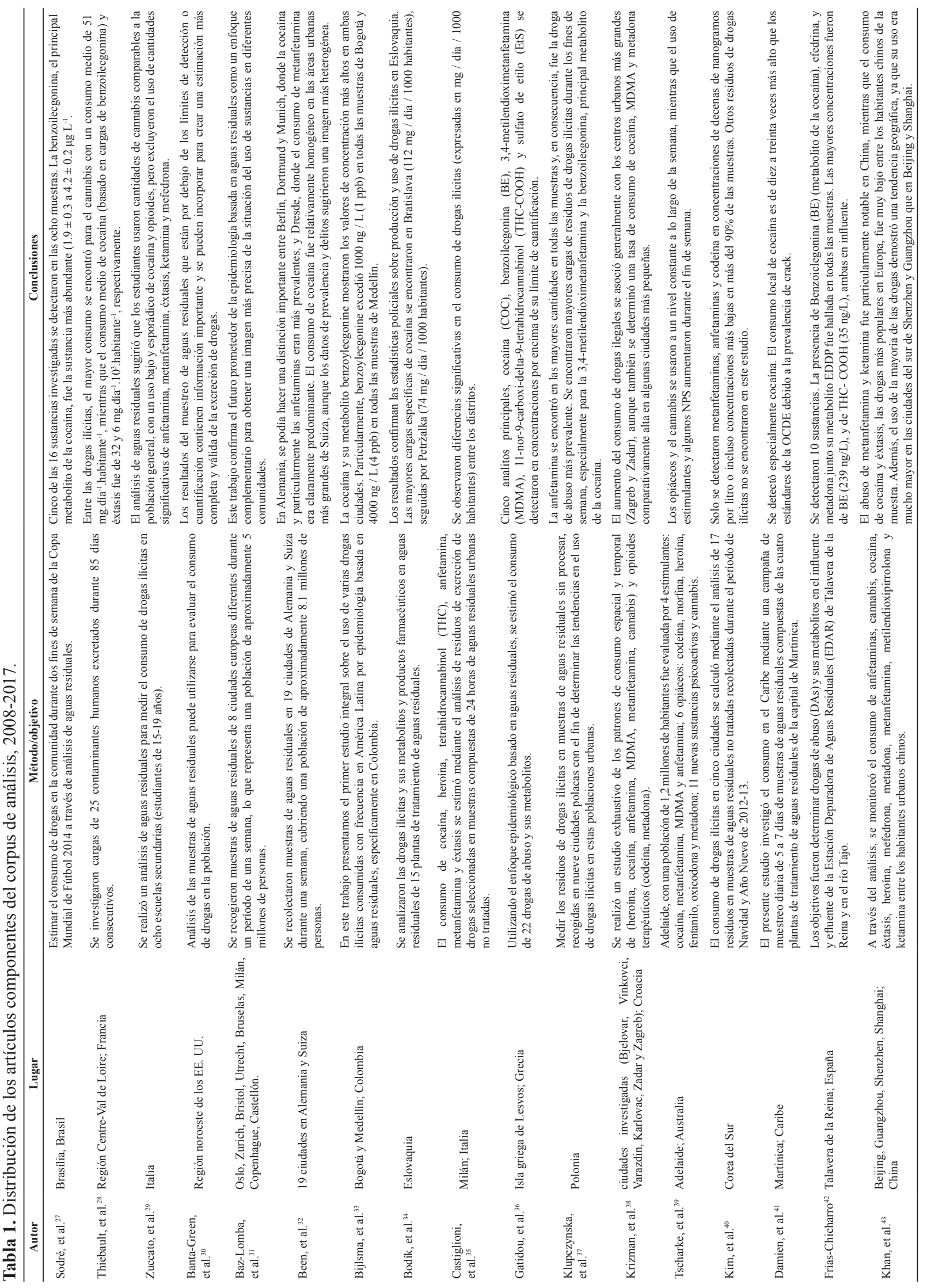




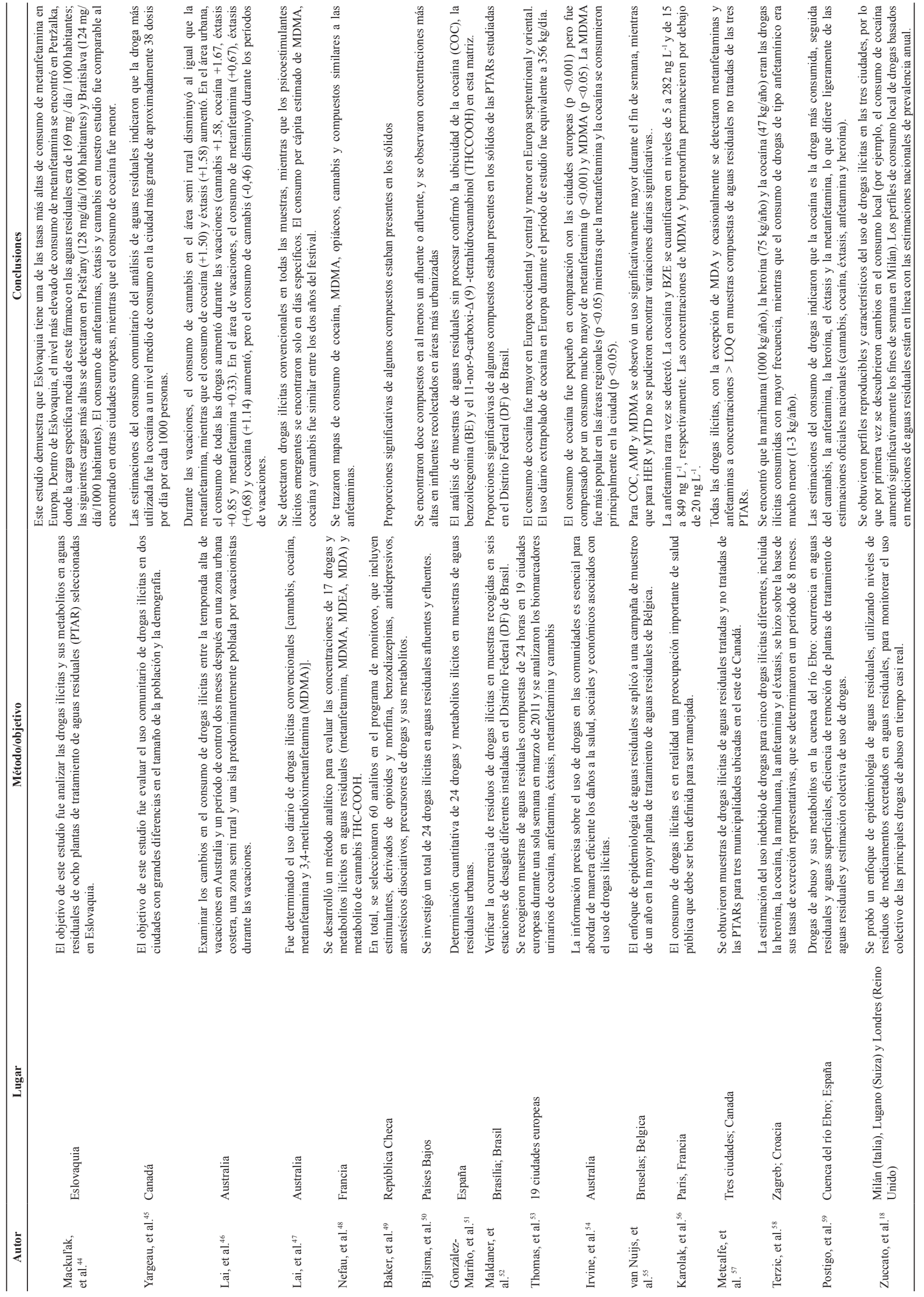


Las descargas de aguas residuales municipales insuficientemente tratadas son la ruta principal para la contaminación de aguas superficiales. La contaminación por drogas de abuso en concentraciones muy bajas parece ser amplia en compartimentos ambientales cerca de áreas pobladas, y ha sido demostrada por diversos estudios de campo nacionales e internacionales ${ }^{61,62}$. Aunque las concentraciones informadas son insignificantes para los seres humanos, la situación podría generar riesgos potenciales para la biota acuática que amenaza la estabilidad y diversidad de los servicios del ecosistema ${ }^{63}$.

Numerosas actividades contribuyen al aporte de sustancias químicas en el ambiente. Sin embargo, el descarte de desagüe bruto y de efluentes de PTARs son consideradas fuentes mayoritarias de aporte de numerosos contaminantes emergentes para sistemas acuáticos, incluyendo las drogas de abuso. La Figura 2 muestra las principales vías de transporte de drogas de abuso, así como de sus metabolitos, para el ambiente.

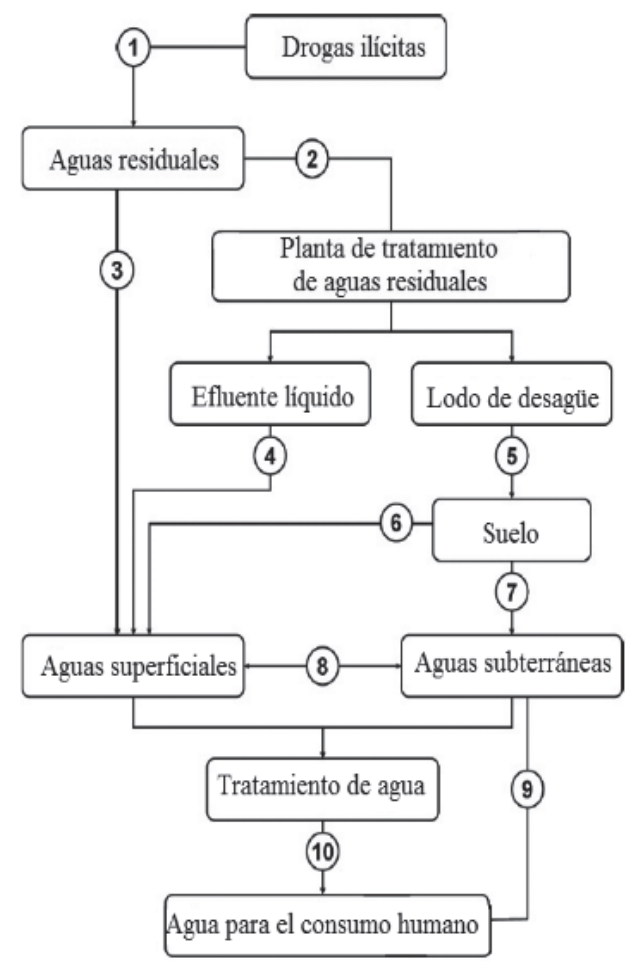

Figura 2. Origen y destino de drogas ilícitas en el ambiente

La ruta 1 representa el enriquecimiento del desagüe bruto con drogas ilícitas a partir de la excreción fecal y/o urinaria de usuarios, además de la contribución del descarte intencional y/o accidental de drogas en sistemas de captación de alcantarillas. Los servicios de alcantarillado sanitario están representados por las rutas 2 y 3 , siendo que la primera corresponde a la canalización del alcantarillado hasta las PTARs y la segunda, al servicio de expulsión del alcantarillado bruto de la población. El descarte clandestino del desagüe también se contempla en la ruta 3 .

Así como en ambientes acuáticos naturales, en las PTARs es posible prever o estimar la fugacidad de una sustancia química, es decir, su permanencia en el efluente líquido o en el lodo generado durante el tiempo tratamiento. Obviamente, durante el proceso de tratamiento, muchas sustancias pueden ser total o parcialmente degradadas o biotransformadas.

Sin embargo, la identificación de algunas propiedades físico-químicas, puede subsidiar acciones o dirigir estudios orientados al diagnóstico de áreas o ambientes acuáticos impactados por la ocurrencia de drogas de abuso. Este conocimiento es notablemente importante ya que el efluente es generalmente descartado en sistemas de aguas superficiales, como señala la ruta 4, mientras que el material sólido puede ser utilizado para la fertilización y/o acondicionamiento de suelos cultivables (ruta 5). Así, la contaminación de aguas naturales por medio del flujo superficial (ruta 6) o de la lixiviación (ruta 7) también puede ocurrir si las drogas de abuso en el lodo de desagüe aplicado al suelo.

La ruta 8 representa la interfaz existente entre los sistemas acuáticos superficiales y subterráneos. El agua subterránea puede ser bombeada y utilizada para fines de consumo (ruta 9) o sometida a tratamiento. Finalmente, la ruta 10 representa la distribución de agua para consumo humano a través de redes municipales de suministro.

El objetivo de la epidemiología de alcantarillado es realizar una estimación de los niveles de consumo de drogas a partir de los análisis de las aguas residuales. Es una metodología relativamente nueva, que ha mostrado resultados prometedores ${ }^{17-19,24}$, donde los investigadores tratan en primer lugar de identificar y cuantificar residuos de drogas y, a continuación, hacen un cálculo retrospectivo para determinar la cantidad de drogas ilegales consumidas por la población atendida por las PTARs ${ }^{22}$. Este proceso consta de varias fases. Inicialmente, se obtienen muestras compuestas de aguas residuales no tratadas de las alcantarillas de una zona geográfica definida. Seguidamente se analizan las muestras para determinar las concentraciones de los residuos de la droga objetivo. A continuación, se estima el consumo de droga mediante un cálculo retrospectivo que consiste en multiplicar la concentración de residuos 
de cada droga objetivo (ng/L) por el correspondiente caudal de aguas residuales (L/día). Como parte de la ecuación, se aplica un factor de corrección para cada droga. El último paso consiste en dividir el resultado por la población atendida por la planta de tratamiento de aguas residuales, para obtener la cantidad de sustancia consumida al día por 1.000 habitantes.

\section{Discusión}

En relación a la detección de drogas ilícitas y metabólitos en PTARs en el contexto de la epidemiología de alcantarillado, la investigación evidenció un uso diseminado de psicotrópicos. Además de los problemas notorios referentes al consumo abusivo, nuevas evidencias han revelado una faceta ambiental frente a la presencia de estas sustancias en el día a día de grandes ciudades. Las drogas ilícitas de diferentes clases, así como sus productos de metabolización y/o biotransformación, se han encontrado recientemente en muestras de diferentes compartimentos ambientales. La presencia de estas sustancias en aguas naturales implica en condiciones inadecuadas de gestión de residuos urbanos, una vez que el desagüe bruto surge como vector mayoritario de contaminación de aguas, así como ocurre para otros contaminantes emergentes.

Aún se sabe poco sobre la ecotoxicidad de estos contaminantes y menos aún sobre un posible cuadro de exposición crónica de organismos en desarrollo, incluidos los seres humanos, frente al agua que contiene trazas de drogas ilícitas y metabolitos. Por otro lado, la ocurrencia de estas sustancias en el alcantarillado bruto ha servido como herramienta para estimar el consumo de una población, visto que, generalmente, la obtención de información confiable sobre tráfico y el consumo de drogas son subjetivo y limitado por la propia naturaleza ilícita de la actividad. Este nuevo enfoque forense, denominado epidemiología del alcantarillado, avanza a pasos agigantados para consolidarse como una poderosa herramienta para obtener información en tiempo real sobre el consumo y la producción de drogas, sumándose a los métodos tradicionales de recolección de datos. Las posibilidades de aplicación de una nueva herramienta son innumerables y sólo la contribución de la toxicología, junto con disciplinas relacionadas, permitirá que la epidemiología del alcantarillado tenga todo su potencial explotado.

Es importante comprender mejor el alcance del uso de drogas ilícitas para los políticos que buscan obstaculizar las actividades delictivas y sus ingresos.
Esto es particularmente importante con respecto a las discusiones sobre la legalización de uso y la regulación de los mercados ${ }^{64}$.

Los reflejos de las polémicas que involucran el uso de drogas y la falta de una política pública integrada hacen percibir que los órganos gubernamentales, la mayoría de las veces, actúan aisladamente, y difícilmente logran traducir sus propuestas en acciones prácticas ${ }^{64}$. De esta manera, aunque son relativamente frecuentes los discursos políticos, las conferencias, los simposios e incluso los encuentros científicos sobre el tema, son pocas las intervenciones preventivas implementadas de hecho. En este contexto, las propuestas gubernamentales que lograron alcanzar un estadio más avanzado de implementación, se debilitaron en el proceso de cambio de gobierno, o incluso acabaron siendo totalmente sustituidas sin ningún fundamento científico, lógico y práctico.

En América latina, pocos estudios han sido desarrollados con el propósito de estimar el consumo de drogas ilícitas que permita trazar el perfil epidemiológico de la población en cuanto al consumo. En realidad, los países se resienten de la ausencia de un sistema de información capaz de contemplar las diversidades regionales y de producir políticas locales orientadas por políticas nacionales. No se ha observado crecimiento de la producción científica en esta área, particularmente en las últimas dos décadas.

\section{Conclusiones}

La presencia de drogas de abuso en el ambiente plantea una preocupación creciente debido a sus efectos adversos ya bien demostrados en la población. La naturaleza y cantidad están frecuentemente relacionados con las características, estado de salud y hábitos de las poblaciones conectadas a las PTARs. Por todo ello, el estudio de estos contaminantes tiene una doble vertiente: epidemiológicamente se pueden evaluar la magnitud, naturaleza y patrones de consumo a través del abanico de compuestos detectados en las PTARs y ambientalmente se puede seguir el rastro y gestionar el peligro que estas sustancias representan para los ecosistemas.

Al abarcar las concepciones de detección de drogas ilícitas en el alcantarillado sanitario, la presente revisión demuestra que los avances en las legislaciones y políticas nacionales, por importantes que sean, son limitados para modificar las visiones sobre el tema, sobre todo al uso. Sin embargo, a través del conocimiento 
de esas concepciones, se puede iniciar el debate que busca considerar a los actores que forman parte de esos contextos. Existe la tendencia de mirar al usuario de drogas descolgado de la sociedad en la que se inserta. Por más que se espere que el proceso de formación y el papel que ocupan traigan sustratos importantes para una visión más integral y contextualizada sobre las consecuencias del uso de drogas, sus entendimientos sobre determinados asuntos son atravesados por valores societarios, con gran influencia del poder económico.

Por fin cabe destacar que, a nivel mundial, la dependencia de drogas ilícitas y no registradas sigue siendo una fuente clave de morbosidad y mortalidad, y está implicada en la incidencia criminal.

\section{Conflictos de interés}

Lo autor no tienen conflictos de intereses que declarar.

\section{Agradecimiento}

Conselho Nacional de Desenvolvimento Científico e Tecnológico (CNPq).

\section{Referencias}

1. Bucher R. As drogas e a vida: uma abordagem biopsicossocial. São Paulo: EPU; 1988.

2. Hebdige D. The Subcultural Meaning of Style. Londres: Routledge; 2002.

3. Petrie B, Barden R, Kasprzyk-Hordern B. A review on emerging contaminants in wastewaters and the environment: Current knowledge, understudied areas and recommendations for future monitoring. Water Res. 2015; 72: 3-27. doi: 10.1016/j. watres.2014.08.053.

4. Rosi-Marshall EJ, Snow D, Bartelt-Hunt SL, Paspalof A, Tank JL. A review of ecological effects and environmental fate of illicit drugs in aquatic ecosystems. J Hazard Mater. 2015; 282: 18-25. doi: 10.1016/j.jhazmat.2014.06.062.

5. Pal R, Megharaj M, Kirkbride KP, Naidu R. Illicit drugs and the environment - A review. Sci Total Environ. 2013; 463-464: 1079-1092. doi: 0.1016/j. scitotenv.2012.05.086.

6. Moore LD, Elkavich A. Who's using and who's doing time: incarceration, the war on drugs, and public health. Am J Public Health. 2008; 98(5): 782786. doi: 10.2105/AJPH.2007.126284.

7. Whiteford HA, Degenhardt L, Rehm J, Baxter AJ, Ferrari AJ, Erskine HE, et al. Global burden of disease attributable to mental and substance use disorders: findings from the Global Burden of Disease Study 2010. The Lancet. 2013; 382(9904): 1575-1586. doi: 10.1016/S0140-6736(13)61611-6.

8. Jimenez Herrera L. Prevention against drugs. Rev Costarric Salud Públ. 2010; 19(2): 93-100.

9. González-López JR, Rodríguez-Gázquez MA, Lomas-Campos MM. Prevalencia de consumo de alcohol, tabaco y drogas ilícitas en inmigrantes latinoamericanos adultos. Rev Latino-Americana Enferm. 2012; 20(3): 528-535. doi: 10.1590/S010411692012000300014.

10. Córdoba D, Toledo D. Cocaína y base de cocaína. Basuco. En: Córdoba D. Bogotá "Toxicología". Bogotá: ed Editorial El Manual Moderno; 2001.

11. Ribeiro M, Marques ACPR, Laranjeira R, Alves HNP, Araújo MR, Baltieri DA, et al. Abuso e dependência da maconha. Rev. Assoc. Med. Bras. 2005; 51(5): 247-249. doi: 10.1590/S010442302005000500008.

12. Ballani TSL, Oliveira MLF. Use of drugs of abuse and sentinel event: constructing a proposition about assessing public policies. Texto Contexto Enferm. 2007; 16(3): 488-494. doi: 10.1590/S010407072007000300015.

13. Bernal E. ¿Tienen futuro las guías de práctica clínica en el Sistema Nacional de Salud? Gestión Clín Sanit. 2002; 4(3): 75-77.

14. Sanchez Z, Oliveira LG, Nappo SA. Main reasons for non-use of illicit drugs by young population exposed to risk situations. Rev Saude Publica. 2005;39(4):17. doi: 10.1590/S0034-89102005000400013.

15. Caiaffa WT, Bastos FI, Freitas LL, Mingoti SA, Proietti FA, Carneiro-Proietti AB, et al. The contribution of two Brazilian multi-center studies to the assessment of HIV and HCV infection and prevention strategies among injecting drug users: The Ajude-Brasil I and II Projects. Cad Saude Publica. 2006; 22(4): 771-782. doi: 10.1590/S0102311 X2006000400016.

16. Alves VS. Health care models for users of alcohol and other drugs: political discourse, knowledge and practices. Cad Saude Publica. 2009; 25(11): 23092319. doi: 10.1590/S0102-311X2009001100002.

17. Zuccato E, Chiabrando C, Castiglioni S, Calamari $\mathrm{D}$, Bagnati R, Schiarea S, et al. Cocaine in surface water: a new evidence based tool to monitor community drug abuse. Environ. Health: A Global Access Science Source. 2005; 4:14. doi: 10.1186/1476-069X-4-14.

18. Zuccato E, Chiabrando C, Castigliano S, Bagnati R, Fanelli R. Estimating community drug abuse by wastewater analysis. Environ. Health Perspect. 2008; 116(8): 1027-1032. doi. 10.1289/ehp.11022. 
19. Zuccato E, Castiglioni S, Bagnati R, Chiabrando C, Grassi P, Fanelli R. Illicit drugs, a novel group of environmental contaminants. Water Res. 2008; 42(45): 961-868. doi: 10.1016/j.watres.2007.09.010.

20. Gil MJ, Soto AM, Usma JI, Gutiérrez OD. Contaminantes emergentes en aguas, efectos y posibles tratamientos. Producc Limpia. 2012; 7(2): 52-73.

21. Tejada C, Quiñonez E, Peña M. Contaminantes emergentes en aguas: metabolitos de fármacos. una revisión. Rev Fac Cienc Básicas. 2014; 10(1): 80-101.

22. Castiglioni S, Thomas KV, Kasprzyk-Hordern B, Vandam L, Griffiths P. Testing wastewater to detect illicit drugs: State of the art, potential and research needs. Sci Total Environ. 2014; 487: 613-620. doi: 10.1016/j.scitotenv.2013.10.034.

23. Castiglioni S, Bijlsma L, Covaci A, Emke E, Hernández F, Reid M, et al. Evaluation of uncertainties associated with the determination of community drug use through the measurement of sewage drug biomarkers. Environ Sci Technol. 2013; 47(3): 1452-1460. doi: 10.1021/es302722f.

24. EMCDDA. Assessing illicit drugs in wastewater: advances in wastewater-based drug epidemiology. EMCDDA Insights 22. Luxembourg: Publications Office of the European Union; 2016. doi: 10.2810/017397.

25. Sampaio RF, Mancini MC. Estudos de revisão sistemática: um guia para síntese criteriosa da evidência científica. Braz J Phys Ther. 2007; 11(1): 83-89. doi: 10.1590/S1413-35552007000100013.

26. Moher D, Liberati A, Tetzlaff J, Altman DG. PRISMA Group. Preferred reporting items for systematic reviews and meta-analyses: the PRISMA statement. PLoS Med. 2009; 6: e1000097.

27. Sodré FF, Souza GB, Feitosa RS, Pereira CEB, Maldaner AO. Illicit Drugs, Metabolites and adulterants in wastewater: monitoring community drug abuse in the Brazilian Federal District during the 2014 Soccer World Cup. J Braz Chem Soc. 2017; 28(11): 2146-2154. doi: 10.21577/01035053.20170063 .

28. Thiebault T, Fougère L, Destandau E, Réty M, Jacob J. Temporal dynamics of human-excreted pollutants in wastewater treatment plant influents: Toward a better knowledge of mass load fluctuations. Sci Total Environ. 2017; 596-597: 246-255. doi: 10.1016/j. scitotenv.2017.04.130.

29. Zuccato E, Gracia-Lor E, Rousis NI, Parabiaghi A, Senta I, Riva F, Castiglioni S. Illicit drug consumption in school populations measured by wastewater analysis. Drug Alcohol Depend. 2017; 178: 285-
290. doi: 10.1016/j.drugalcdep.2017.05.030.

30. Banta-Green CJ, Brewer AJ, Ort C, Helsel DR, Williams JR, Field JA. Using wastewater-based epidemiology to estimate drug consumptionStatistical analyses and data presentation. Sci Total Environ. 2016; 568: 856-863. doi: 10.1016/j. scitotenv.2016.06.052.

31. Baz-Lomba JA, Salvatore S, Gracia-Lor E, Bade $\mathrm{R}$, Castiglioni S, Castrignanò $\mathrm{E}$, et al. Comparison of pharmaceutical, illicit drug, alcohol, nicotine and caffeine levels in wastewater with sale, seizure and consumption data for 8 European cities. BMC Public Health. 2016; 16(1): 1035. doi: 10.1186/ s12889-016-3686-5.

32. Been F, Bijlsma L, Benaglia L, Berset JD, Botero-Coy AM, Castiglioni S, et al. Assessing geographical differences in illicit drug consumption -A comparison of results from epidemiological and wastewater data in Germany and Switzerland. Drug Alcohol Depend. 2016; 161: 189-199. doi: 10.1016/j.drugalcdep.2016.02.002.

33. Bijlsma L, Botero-Coy AM, Rincón RJ, Peñuela GA, Hernández F. Estimation of illicit drug use in the main cities of Colombia by means of urban wastewater analysis. Sci Total Environ. 2016; 565: 984-993. doi: 10.1016/j.scitotenv.2016.05.078.

34. Bodík I, Mackul'ak T, Fáberová M, Ivanová L. Occurrence of illicit drugs and selected pharmaceuticals in Slovak municipal wastewater. Environ Sci Pollut Res Int. 2016; 23(20): 2109821105. doi: 10.1007/s11356-016-7415-5.

35. Castiglioni S, Borsotti A, Riva F, Zuccato E. Illicit drug consumption estimated by wastewater analysis in different districts of Milan: A case study. Drug Alcohol Rev. 2016; 35(2): 128-132. doi: 10.1111/ dar. 12233.

36. Gatidou G, Kinyua J, van Nuijs AL, Gracia-Lor E, Castiglioni S, Covaci A, et al. Drugs of abuse and alcohol consumption among different groups of population on the Greek Island of Lesvos through sewage-based epidemiology. Sci Total Environ. 2016;563-564:633-640. DOI: 10.1016/j. scitotenv.2016.04.130.

37. Klupczynska A, Dereziński P, Krysztofiak J, Kokot ZJ. Estimation of drug abuse in 9 Polish cities by wastewater analysis. Forensic Sci Int. 2016; 260: 14-21. doi: 10.1016/j.forsciint.2015.12.042.

38. Krizman I, Senta I, Ahel M, Terzic S. Wastewaterbased assessment of regional and temporal consumption patterns of illicit drugs and therapeutic opioids in Croatia. Sci Total Environ. 2016; 566567: 454-462. doi: 10.1016/j.scitotenv.2016.05.075. 39. Tscharke BJ, Chen C, Gerber JP, White JM. Temporal 
trends in drug use in Adelaide, South Australia by wastewater analysis. Sci Total Environ. 2016; 565: 384-391. doi: 10.1016/j.scitotenv.2016.04.183.

40. Kim KY, Lai FY, Kim HY, Thai PK, Mueller JF, Oh JE. The first application of wastewater-based drug epidemiology in five South Korean cities. Sci Total Environ. 2015; 524-525: 440-446. doi: 10.1016/j. scitotenv.2015.04.065.

41. Damien DA, Thomas N, Hélène P, Sara K, Yves L. First evaluation of illicit and licit drug consumption based on wastewater analysis in Fort de France urban area (Martinique, Caribbean), a transit area for drug smuggling. Sci Total Environ. 2014; 490: 970-978. doi: 10.1016/j.scitotenv.2014.05.090.

42. Frías-Chicharro A, González-Alonso S, MonteroRubio JC, Valcárcel-Rivera Y. Estimación del consumo de drogas de abuso y sus metabolitos a partir de su presencia en el agua residual de Talavera de la Reina y en el río Tajo. Rev Esp Salud Publ. 2014; 88(2): 289-299. doi: 10.4321/S113557272014000200011.

43. Kankaanpää A, Ariniemi K, Heinonen M, Kuoppasalmi K, Gunnar T. Use of illicit stimulant drugs in Finland: a wastewater study in ten major cities. Sci Total Environ. 2014; 487: 696-702. doi: 10.1016/j.scitotenv.2013.11.095.

44. Mackul'ak T, Skubák J, Grabic R, Ryba J, Birošová L, Fedorova G, et al. National study of illicit drug use in Slovakia based on wastewater analysis. Sci Total Environ. 2014; 494-495: 158-165. doi: 10.1016/j.scitotenv.2014.06.089.

45. Yargeau V, Taylor B, Li H, Rodayan A, Metcalfe CD. Analysis of drugs of abuse in wastewater from two Canadian cities. Sci Total Environ. 2014; 487: 722-730. doi: 10.1016/j.scitotenv.2013.11.094.

46. Lai FY, Bruno R, Hall W, Gartner C, Ort C, Kirkbride $\mathrm{P}$, et al. Profiles of illicit drug use during annual key holiday and control periods in Australia: wastewater analysis in an urban, a semi-rural and a vacation area. Addiction. 2013; 108(3): 556-565. doi: 10.1111/add.12006.

47. Lai FY, Thai PK, O'Brien J, Gartner C, Bruno $\mathrm{R}$, Kele $\mathrm{B}$, et al. Using quantitative wastewater analysis to measure daily usage of conventional and emerging illicit drugs at an annual music festival. Drug Alcohol Rev. 2013; 32(6): 594-602. doi: 10.1111/dar.12061.

48. Nefau T, Karolak S, Castillo L, Boireau V, Levi Y. Presence of illicit drugs and metabolites in influents and effluents of 25 sewage water treatment plants and map of drug consumption in France. Sci Total Environ. 2013; 461-462: 712-722. doi: 10.1016/j. scitotenv.2013.05.038.
49. Baker DR, Očenášková $V$, Kvicalova $M$, Kasprzyk-Hordern B. Drugs of abuse in wastewater and suspended particulate matter further developments in sewage epidemiology. Environ Int. 2012; 48: 28-38. doi: 10.1016/j. envint.2012.06.014.

50. Bijlsma L, Emke E, Hernández F, de Voogt P. Investigation of drugs of abuse and relevant metabolites in Dutch sewage water by liquid chromatography coupled to high resolution mass spectrometry. Chemosphere. 2012; 89(11): 13991406. doi: 10.1016/j.chemosphere.2012.05.110.

51. González-Mariño I, Quintana JB, Rodríguez I, González-Díez M, Cela R. Screening and selective quantification of illicit drugs in wastewater by mixed-mode solid-phase extraction and quadrupole-time-of-flight liquid chromatographymass spectrometry. Anal Chem. 2012; 84(3): 17081717. doi: $10.1021 /$ ac202989e.

52. Maldaner AO, Schmidt LL, Locatelli MAF, Jardim WF, Sodré FF, Almeida FV, et al. Estimating cocaine consumption in the Brazilian Federal District (FD) by sewage analysis. Braz Chem Soc. 2012; 23(5), 861-867. doi: 10.1590/S0103-50532012000500011.

53. Thomas KV, Bijlsma L, Castiglioni S, Covaci A, Emke E, Grabic R, et al. Comparing illicit drug use in 19 European cities through sewage analysis. Sci Total Environ. 2012; 432: 432-439. doi: 10.1016/j. scitotenv.2012.06.069.

54. Irvine RJ, Kostakis C, Felgate PD, Jaehne EJ, Chen C, White JM. Population drug use in Australia: a wastewater analysis. Forensic Sci Int. 2011; 210(13): 69-73. doi: 10.1016/j.forsciint.2011.01.037.

55. van Nuijs AL, Mougel JF, Tarcomnicu I, Bervoets L, Blust R, Jorens PG, et al. Sewage epidemiology a real-time approach to estimate the consumption of illicit drugs in Brussels, Belgium. Environ Int. 2011; 37(3): 612-621. doi: 10.1016/j.envint.2010.12.006.

56. Karolak S, Nefau T, Bailly E, Solgadi A, Levi Y. Estimation of illicit drugs consumption by wastewater analysis in Paris area (France). Forensic Sci Int. 2010; 200(1-3): 153-160. doi: 10.1016/j. forsciint.2010.04.007.

57. Metcalfe C, Tindale K, Li H, Rodayan A, Yargeau V. Illicit drugs in Canadian municipal wastewater and estimates of community drug use. Environ Pollut. 2010; 158(10): 3179-3185. doi: 10.1016/j. envpol.2010.07.002.

58. Terzic S, Senta I, Ahel M. Illicit drugs in wastewater of the city of Zagreb (Croatia) - estimation of drug abuse in a transition country. Environ Pollut. 2010; 158(8): 2686-2693. doi: 10.1016/j. envpol.2010.04.020. 
59. Postigo C, López de Alda MJ, Barceló D. Drugs of abuse and their metabolites in the Ebro River basin: occurrence in sewage and surface water, sewage treatment plants removal efficiency, and collective drug usage estimation. Environ Int. 2009; 36(1): 7584. doi: 10.1016/j.envint.2009.10.004.

60. Viana M, Querol X, Alastuey A, Postigo C, de Alda MJ, Barceló D, et al. Drugs of abuse in airborne particulates in urban environments. Environ Int. 2010; 36(6): 527-534. doi: 10.1016/j. envint.2010.04.004.

61. Robles-Molina J, Gilbert-López B, García-Reyes JF, Molina-Díaz A. Monitoring of selected priority and emerging contaminants in the Guadalquivir River and other related surface waters in the province of Jaén, South East Spain. Sci Total Environ. 2014; (479-480): 247-257. doi: 10.1016/j. scitotenv.2014.01.121.

62. Jiang JJ, Lee CL, Fang MD, Tu BW, Liang YJ. Impacts of Emerging Contaminants on surrounding aquatic environment from a youth festival. Environ Sci Technol. 2015; 49(2): 792-799. doi: 10.1021/ es503944e.

63. Catalá M, Domínguez-Morueco N, Migens A, Molina R, Martínez F, Valcárcel $\mathrm{Y}$, et al. Elimination of drugs of abuse and their toxicity from natural waters by photo-Fenton treatment. Sci Total Environ. 2015; 520: 198-205. doi: 10.1016/j. scitotenv.2015.03.042.

64. Laranjeira R. Legalização de drogas e a saúde pública. Ciênc. saúde coletiva. 2010; 15(3): 621631. doi: 10.1590/S1413-81232010000300002. 